\title{
Liquid Chromatography-Mass Spectrometry in the Analysis of Pesticide Residues in Food
}

\author{
Anna Stachniuk ${ }^{1} \cdot$ Emilia Fornal $^{1}$ \\ Received: 13 July 2015 / Accepted: 18 October 2015 /Published online: 28 October 2015 \\ (C) The Author(s) 2015. This article is published with open access at Springerlink.com
}

\begin{abstract}
The analysis of pesticide residues in food is nowadays an increasingly important task. Quality control has to be very strict in order to safeguard the consumers' health. One of the most important goals of food quality assurance is testing food for residues and contaminants. Among chemical hazards, the contamination of food with pesticides has been characterized as a significant source of many serious diseases. Consumption of food containing pesticide residues may cause cancer, malformations, and damage to the endocrine, nervous, and immune system. In order to assure human food safety, The European Community has established maximum residue limits (MRL) of pesticides permitted in products of animal or vegetable origin that are intended for human consumption. In the EU, Regulation (EC) No. 396/2005 of the European Parliament and Council on pesticide residues established the levels of pesticide residues allowed in food and feed. To ensure the compliance of marketed food commodities with the law on food safety, sensitive and reliable analytical methods for the determination of pesticide residues are required. Multiresidue liquid chromatography-mass spectrometry methods (LC-MS) are widely recognized as an ideal, highly specific, and extremely sensitive technique for testing food products. This review discusses LC-MS approaches applied most widely to pesticide residue analysis over the last few years. The main ionization methods and MS detectors used as analytical tools in quantification and/or identification/confirmation of pesticide residues in food are presented.
\end{abstract}

Emilia Fornal

efornal@kul.pl

1 Laboratory of Separation and Spectroscopic Method Applications, Center for Interdisciplinary Research, The John Paul II Catholic University of Lublin, Konstantynów 1F, 20-708 Lublin, Poland
Keywords Pesticide residues $\cdot$ Liquid chromatography-mass spectrometry $\cdot$ Fruits and vegetables $\cdot$ Food safety

\section{Introduction}

The control of pesticide residues in food is required by law to assure human food safety and safeguard the consumers' health. In Europe, maximum residue limits (MRL) of pesticides permitted in products of animal or vegetable origin that are intended for human consumption have been established by Regulation (EC) No. 396/2005 of the European Parliament and Council on pesticide residues. Nowadays, gas chromatography coupled to mass spectrometry (GC-MS, GC-MS/MS) with electron impact ionization (EI) and liquid chromatography-tandem mass spectrometry (LC-MS/MS) with electrospray ionization (ESI) are techniques most often employed for multiresidue pesticide analysis in food due to their high sensitivity and selectivity, ability to screen many pesticides from various chemical classes in very complex matrixes in a single run. The former may be used only for the analysis of volatile chemicals; thus, the analyzed pesticide has to be volatile or amenable to derivatization to ensure its volatility. GC-MS is a method of choice for less polar pesticides; for more polar compounds, LC-MS is more suitable. The need to deal with more polar pesticides as well as with pesticide metabolites, which are often more polar and less volatile than pesticide itself, is one of the main reasons for choosing LCMS/MS over GC-MS. Compounds which are thermolabile, not volatile, and have not been derivatized can be separated by LC-MS. LC-MS can analyze a much wider range of chemicals than GC-MS. The thorough discussion on application of GC-MS, GC-MS/MS, and LC-MS/MS for the analysis of different chemical classes of pesticides can be found in the book chapter by Raina (2011). The assessment for the 
evaluation of the capabilities of GC-MS(/MS) and LCMS/MS for the determination of pesticides carried out by Alder et al. (2006) and Carmona et al. (2013) showed that a wider scope and better sensitivity is achieved by LC-MS.

The wide scope of pesticides covered and simple sample preparation is the key reason why liquid chromatography coupled to mass spectrometry is more and more frequently used for the detection, identification, and quantification of pesticides in food nowadays. This technique provides information about the structure of the analyte, without the need to derivatize the analyte. Its sample purity requirements are not stringent, and it enables a simultaneous analysis of substances that vary considerably in polarity. The popularity of the method is confirmed by the increasing number of publications dedicated to the applications of LC-MS in the determination of contaminants, including pesticides, in food (Sivaperumal et al. 2015; GómezPérez et al. 2015; Martinez-Dominguez et al. 2015; Golge and Kebak 2015; Fillatre et al. 2014; Esturk et al. 2014; Oshita and Jardim 2014; González-Curbelo et al. 2014; Rajski et al. 2013; Arienzo et al. 2013; Stachniuk and Fornal 2013; Sinha et al. 2012; Gilbert-Lopez et al. 2012; Lozano et al. 2012; Chung and Lam 2012; BoteroCoy et al. 2012; Tian 2011; Pareja et al. 2011; Kmellar et al. 2011; Aguilera-Luiz et al. 2011; Chen et al. 2011; Lehotay et al. 2010; Zhang et al. 2010; Camino-Sanchez et al. 2010; Kamel et al. 2010; Brutti et al. 2010). In pesticide residue analysis, similarly to the analysis of other food contaminants, there is a distinct tendency to develop fast multiresidue methods.

In a typical LC-MS system, the sample analyzed is initially separated in the LC system and its successive fractions eluted from the chromatographic column are subjected to ionization and introduced into the spectrometer. Nowadays high-performance liquid chromatography (HPLC) and ultra high-performance liquid chromatography (UHPLC) are usually employed for the separation of analytes.

The paper presents the elements of the LC-MS systems and discusses mobile phases, sorbents, types of MS analyzers, and ion detection techniques most frequently used in the analysis of pesticide residues in food. Besides the advantages of using LC-MS systems, the limitations of the method, resulting mainly from the matrix effect, and the means of minimizing its impact are discussed.

\section{Liquid Chromatography}

Most of the procedures used to determine a considerable number of pesticides in food, as described in the literature, employ the HPLC technique in a reversed-phase system, using gradient elution with a linear increase of the percentage of the organic solvent. Isocratic elution is used sporadically (Chung and Lam 2012). The advantage of gradient elution is the possibility of achieving the optimum conditions of separation by decreasing the polarity of the mobile phase. This leads to increased eluting strength and elution of hydrophobic substances, strongly interacting with the stationary phase, from the column. The hydrophilic molecules leave the column first, followed by molecules with a lower polarity and, finally, hydrophobic molecules. Mixtures of acetonitrile, methanol, and water in various volume ratios are used as eluents. Separation efficiency is further enhanced by adding an organic acid (formic or acetic), ammonium salts (ammonium formate or ammonium acetate), or a combination of an organic acid with its ammonium salt, to the mobile phase. The additives to $\mathrm{LC} /$ MS mobile phases are restricted to volatile compounds; nonvolatile compounds such as phosphorous salts have to be avoided. The non-polar octadecylsilane phase $\left(\mathrm{C}_{18}\right)$ (Gómez-Pérez et al. 2015; Fillatre et al. 2014; AguileraLuiz et al. 2011; Chung and Lam 2012; Gilbert-Lopez et al. 2012; Sinha et al. 2012) is commonly used as the stationary phase; the medium-polar octylsilane phase is used much more rarely $\left(\mathrm{C}_{8}\right)$ (Lozano et al. 2012; Nunez et al. 2012; Kmellar et al. 2011; Ferrer et al. 2011). Columns with sorbent particle diameters of $3,3.5,4$, and $5 \mu \mathrm{m}$ are available from a range of manufacturers, the most popular being: Zorbax (Rajski et al. 2013; Lozano et al. 2012; Lee et al. 2009a, b; Radisic et al. 2009), Atlantis (Tran et al. 2012; Tseng et al. 2009; Romero-Gonzalez et al. 2006), Luna (Camino-Sanchez et al. 2010; Lehotay et al. 2010), Polaris (Anagnostopoulos et al. 2012), and Phenomenex (Fillatre et al. 2014). Short columns with the particle diameter not larger than $2 \mu \mathrm{m}$, first proposed by Waters in 2004 as $\mathrm{BEH} \mathrm{C}_{18}$, with the particle diameter of $1.7 \mu \mathrm{m}$ (Swartz 2005), are gaining increasing popularity. In this group of sorbents, the abovementioned column $\mathrm{BEH} \mathrm{C}_{18}$ (Sivaperumal et al. 2015; Chung and Lam 2012; Chen et al. 2011; Mastovska et al. 2010) and Zorbax $\mathrm{C}_{18}$, with the particle diameter of $1.8 \mu \mathrm{m}$, from Agilent Technology (Perez-Ortega et al. 2012; Stachniuk and Fornal 2013; Sinha et al. 2012), are the most popular in the analysis of pesticide residues in food. Reduced particle diameter necessitates the use of increasingly higher pressures. Therefore, to achieve the sufficient eluent flow rate in the column, it is necessary to use high-pressure pumps dedicated to UHPLC systems.

Thanks to the much greater separation efficiency of the chromatographic system in comparison with conventional HPLC, the UHPLC system is currently an optimum solution for the chromatographic separation of multicomponent mixtures. 


\section{Mass Spectrometry}

Mass spectrometry is a key analytical technique enabling the identification of various groups of chemical compounds, including pesticides. Regardless of its construction and designated use, a mass spectrometer measures the mass-to-charge ratio of charged molecules. Every mass spectrometer consists of three basic components: an ion source, an analyzer where ions are separated according to their mass-to-charge ratio, and a detector where ions are counted. A series connection of several analyzers is the most frequent modification of the system. The analyzer(s) and detector of a mass spectrometer are kept in a high vacuum to avoid accidental collisions with air molecules. Different LC-MS/MS strategies for the analysis of pesticide residues in food are presented in Table 1.

\section{Ionization Methods}

The possibility of ionizing a substance is a basic condition for analyzing it in a mass spectrometer. Ionization methods vary depending on the structure and principle of operation of an ionizer. Electrospray ionization (ESI) and atmospheric pressure chemical ionization (APCI) are the most frequent ionization methods used to determine pesticide residues in food by means of an LC-MS system. Both methods are based on atmospheric pressure ionization (API) enabling the introduction of considerable volumes of solutions flowing out of the chromatographic column into the mass spectrometer without the risk of losing a high vacuum. Both electrospray ionization and atmospheric pressure chemical ionization make it possible to conduct analyses in two operation modes: creation of positive ions and creation of negative ions (Suder and Silberring 2006).

\section{Electrospray Ionization}

Electrospray ionization is a basic method of molecule ionization used in the analysis of multicomponent mixtures in an LC-MS system. It belongs to a group of methods whereby "soft" ionization is carried out under atmospheric pressure. Under this method, the eluate stream leaving the chromatographic column is introduced into the ionization source through the capillary. At the outlet of the capillary, the sample dissolved in the solvent is exposed to a strong nebulizing gas (typically, nitrogen) and a very strong electric field, which results in the atomization of the sample into charged microdroplets. The polarization of the applied electric field determines whether the charge of the microdroplets is positive or negative. The solvent is vaporized from the droplet surface with a stream of dry, heated gas until the ions are desorbed. Electrospray ionization produces charged molecules that are often subjected to further fragmentation in mass analyzers (Johnstone and
Malcolm 2001; Conepts Guide-Agilent 6100 Series Quadrupole LC/MS Systems. Agilent Technologies 2010). The ionization process is shown in Fig. 1.

Electrospray ionization is a highly efficient technique that does not cause the dissociation of the molecules under study. The multiply charged ions generated during the ionization enable the measurement of molecules whose masses exceed the nominal measuring range of the analyzer (Suder and Silberring 2006). Thanks to its wide range of applications, the ESI technique enables the analysis of many kinds of medium- or high-polarity chemical substances whose molecular masses range from 100 to $150,000 \mathrm{Da}$ (Botitsi et al. 2011). The ESI technique also ensures excellent results in the analysis of substances of low molecular mass, particularly if the molecule analyzed demonstrates limited stability during ionization carried out with other techniques. Based on the cited literature, ESI is the most frequently used ion source in the analysis of pesticides in food by means of LC-MS methods (Table 1). The analysis of pesticide residues in food with LC-ESI-MS methods is conducted both in the positive ion mode and negative ion mode.

\section{Atmospheric Pressure Chemical Ionization}

Atmospheric pressure chemical ionization (APCI) is a very similar technique to ESI. The manner of sample ionization is the fundamental difference between the two methods. In APCI, the eluate leaving the chromatographic column is heated and sprayed from the capillary and then captured, in gaseous form, by a stream of gas and carried to the electrode where corona discharges initiate the formation of ions (Johnstone and Malcolm 2001; Concepts Guide-Agilent 6100 Series Quadrupole LC/MS Systems. Agilent Technologies 2010). The ions thus obtained are focused into an analyzer. The ionization process is shown in Fig. 2.

Atmospheric pressure chemical ionization complements the ESI technique. Unlike ESI, it does not produce multiply charged ions, and since the scope of its application requires a higher temperature, it is commonly used to analyze smaller and more thermally stable compounds. The APCI technique enables the analysis of low- or medium-polarity chemical substances whose molecular masses range from 100 to $2000 \mathrm{Da}$ (Botitsi et al. 2011). The APCI ionization technique is used in the analysis of pesticide residues in food mostly in the positive ionization mode, and less frequently than ESI (Table 1).

\section{Kinds of Analyzers}

The analyzer is the most important component determining the performance of a spectrometer. Its task is to separate ions in terms of their mass-to-charge ratio and to direct the beam of focused ions to the detector. Different kinds of analyzers vary 
Table 1 Current LC-MS/MS strategies for the analysis of pesticide residues in food

\begin{tabular}{|c|c|c|c|c|c|c|c|}
\hline No. & Matrix & Number of analytes & Detector ms & Ionization methods & MS mode & $\mathrm{t}_{\mathrm{R}}(*)$ & References \\
\hline 1 & Chicken eggs & 81 & QQQ & ESI & MRM & - & Hildmann et al. 2015 \\
\hline 2 & Baby food & 391 & QQQ & ESI & MRM & + & Gómez-Pérez et al. 2015 \\
\hline 3 & Fruits and vegetables & 60 & TOF & ESI & TIC & + & Sivaperumal et al. 2015 \\
\hline 4 & Nutraceutical products & 99 & QQQ & ESI & SRM & + & Martinez-Dominguez et al. 2015 \\
\hline 5 & Tomatoes & 109 & QQQ & ESI & MRM & + & Golge and Kebak 2015 \\
\hline 6 & Eggs and eggs products & 20 & QQQ & ESI & SRM & + & Choi et al. 2015 \\
\hline 7 & Cocoa beans & 26 & Q-Trap & ESI & MRM & + & Zainudin et al. 2015 \\
\hline 8 & Lavandin oil & 256 & Q-Trap & ESI & SRM & + & Fillatre et al. 2014 \\
\hline 9 & Strawberries & 29 & QQQ & ESI & MRM & + & Oshita and Jardim 2014 \\
\hline 10 & Fruits and vegetables & 43 & QQQ & ESI & MRM & + & González-Curbelo et al. 2014 \\
\hline 11 & Vegetables & 80 & Q-Trap & ESI & MRM & + & Esturk et al. 2014 \\
\hline 12 & Fruits & 46 & Q-Trap & ESI & MRM & + & Jardim et al. 2014 \\
\hline 13 & Honey & 30 & QQQ & ESI & MRM & + & Kujawski et al. 2014 \\
\hline 14 & Cashew & 20 & QQQ & ESI & SRM & + & Silva et al. 2014 \\
\hline 15 & Soft fruits & 56 & QQQ & ESI & MRM & + & Stachniuk and Fornal 2013 \\
\hline 16 & Bananas & 128 & QQQ & ESI & MRM & + & Carneiro et al. 2013 \\
\hline 17 & Vegetables & 14 & QQQ & ESI & MRM & + & Arienzo et al. 2013 \\
\hline 18 & Avocado and almonds & 113 & QQQ & ESI & MRM & + & Rajski et al. 2013 \\
\hline 19 & Fruits and vegetables & 56 & QQQ & ESI & MRM & + & Lee et al. 2013 \\
\hline 20 & Fruits and vegetables & 100 & QQQ & ESI & SRM & + & Nunez et al. 2012 \\
\hline 21 & Tea, chamomile & 86 & QQQ & ESI & SRM & + & Lozano et al. 2012 \\
\hline 22 & Baby food & 116 & QQQ & ESI & MRM & + & Anagnostopoulos et al. 2012 \\
\hline 23 & Wine & 60 & TOF & ESI & TIC & + & Perez-Ortega et al. 2012 \\
\hline 24 & Fruit juices & 174 & Q-Trap & ESI & MRM & + & Tran et al. 2012 \\
\hline 25 & Tropical fruits & 29 & QQQ, Q-TOF & ESI & MRM & + & Botero-Coy et al. 2012 \\
\hline 26 & Different food & 17 & QQQ & ESI, APCI & MRM & + & Chung and Lam 2012 \\
\hline 27 & Fruit beverages & 30 & TOF & ESI & TIC & + & Gilbert-Lopez et al. 2012 \\
\hline 28 & Vegetables & 18 & Q-Trap & ESI & MRM & + & Sinha et al. 2012 \\
\hline 29 & Milk & 29 & IT & ESI & MRM & + & Tian 2011 \\
\hline 30 & Paprika & 168 & QQQ & ESI & MRM & + & Lee et al. 2011 \\
\hline 31 & Fish muscle & 13 & Q-Trap & ESI & MRM & + & Lazartigues et al. 2011 \\
\hline 32 & Tea & 65 & QQQ & ESI & MRM & + & Chen et al. 2011 \\
\hline 33 & Milk & 42 & QQQ & ESI & MRM & + & Aguilera-Luiz et al. 2011 \\
\hline 34 & Fruits and vegetables & 150 & QQQ & ESI & SRM & + & Kmellar et al. 2011 \\
\hline 35 & Fruit juices & 53 & Q-Trap & ESI & SRM & + & Ferrer et al. 2011 \\
\hline 36 & Tea & 39 & Q & ESI & MRM & + & Zhang et al. 2010 \\
\hline 37 & Cereal grains & 68 & QQQ & ESI & MRM & + & Mastovska et al. 2010 \\
\hline 38 & Different food & 9 & IT & APCI & MRM & + & Brutti et al. 2010 \\
\hline 39 & Fruits and vegetables & 14 & QQQ & ESI & SRM & + & Lehotay et al. 2010 \\
\hline 40 & Fruits and vegetables & 11 & QQQ, Q-TOF & ESI & SRM & + & Grimalt et al. 2010 \\
\hline 41 & Grapes & 150 & Q-Trap & ESI & MRM & + & Afify et al. 2010 \\
\hline 42 & Fruits and vegetables & 69 & QQQ & ESI & MRM & + & Camino-Sanchez et al. 2010 \\
\hline 43 & Wine & 46 & QQQ & ESI & SRM & + & Economou et al. 2009 \\
\hline 44 & Fruits and vegetables & 30 & QQQ, Q-TOF & ESI, APCI & MRM & + & Pico et al. 2009 \\
\hline 45 & Fruits and vegetables & 300 & TOF & ESI & MRM & + & Mezcua et al. 2009 \\
\hline 46 & Baby food & 138 & Q-TOF & ESI & MRM & + & Wang and Leung 2009 \\
\hline 47 & Fruits and vegetables & 81 & QQQ & ESI & MRM & + & Tseng et al. 2009 \\
\hline
\end{tabular}


Table 1 (continued)

\begin{tabular}{|c|c|c|c|c|c|c|c|}
\hline No. & Matrix & Number of analytes & Detector ms & Ionization methods & MS mode & $\mathrm{t}_{\mathrm{R}}(*)$ & References \\
\hline 48 & Fruit juices & 12 & IT & ESI & SRM & + & Radisic et al. 2009 \\
\hline 49 & Vegetables & 44 & Q-Trap & ESI & MRM & + & Lee et al. 2009a \\
\hline 50 & Rice & 47 & Q-Trap & ESI & MRM & + & Lee et al. $2009 \mathrm{~b}$ \\
\hline 51 & Strawberry & 100 & QQQ, TOF & ESI & MRM & + & Taylor et al. 2008 \\
\hline 52 & Soft drinks & 100 & TOF & ESI & MRM & + & Garcia-Reyes et al. 2008 \\
\hline 53 & Fruits and vegetables & 46 & IT & ESI & SRM & - & Lesueur et al. 2008 \\
\hline 54 & Honey, meat, eggs & 136 & QQQ, TOF & ESI & MRM & + & Mol et al. 2008 \\
\hline 55 & Fruits and vegetables & 160 & QQQ & ESI & MRM SRM & + & Kmellar et al. 2008 \\
\hline 56 & Fruits & 12 & QQQ, IT, Q-TOF & ESI & MRM & + & Soler et al. 2007 \\
\hline 57 & Fruits and vegetables & 183 & Q-Trap & ESI & MRM & + & Pihlstrom et al. 2007 \\
\hline 58 & Soya & 169 & QQQ & ESI & MRM & + & Pizzutti et al. 2007 \\
\hline 59 & Grapes & 82 & Q-Trap & ESI & MRM & + & Banerjee et al. 2007 \\
\hline 60 & Fruits and vegetables & 15 & IT & ESI & MRM & + & Wang et al. 2007 \\
\hline 61 & Fruits and vegetables & 43 & QQQ & ESI & SRM & + & Hernandez et al. 2006 \\
\hline 62 & Fruits & 10 & Q & APCI & SIM & + & Blasco et al. 2006 \\
\hline 63 & Vegetables & 23 & Q & ESI & SIM & + & Romero-Gonzalez et al. 2006 \\
\hline 64 & Beer and malt & 277 & QQQ & ESI & MRM & + & Omate et al. 2006 \\
\hline 65 & Milk & 30 & QQQ & ESI & MRM & + & Bogialli et al. 2006 \\
\hline 66 & Fruits and vegetables & 14 & Q & ESI & SIM & + & Liu et al. 2005 \\
\hline 67 & Wine & 9 & Q & APCI & SIM & - & Nozal et al. 2005 \\
\hline 68 & Fruits and vegetables & 15 & QQQ & ESI & SIM & + & Lehotay et al. 2005 \\
\hline 69 & Vegetables & 31 & Q & ESI & SIM & + & Frenich et al. 2005 \\
\hline 70 & Fruits and vegetables & 7 & QQQ & ESI & MRM & + & Sannino and Bandini 2005 \\
\hline
\end{tabular}

$t_{R}$ retention time, + or - information about presentation of retention times in the original text of the publication

in terms of characteristics and operation. The key performance parameters of an analyzer include separation efficiency, $\mathrm{m} / \mathrm{z}$ measurement precision, and range of the $\mathrm{m} / \mathrm{z}$ values measured. The choice of a particular analyzer is determined by the kind of analysis conducted. In the multiresidue analysis of pesticides in food, the following analyzers are used most

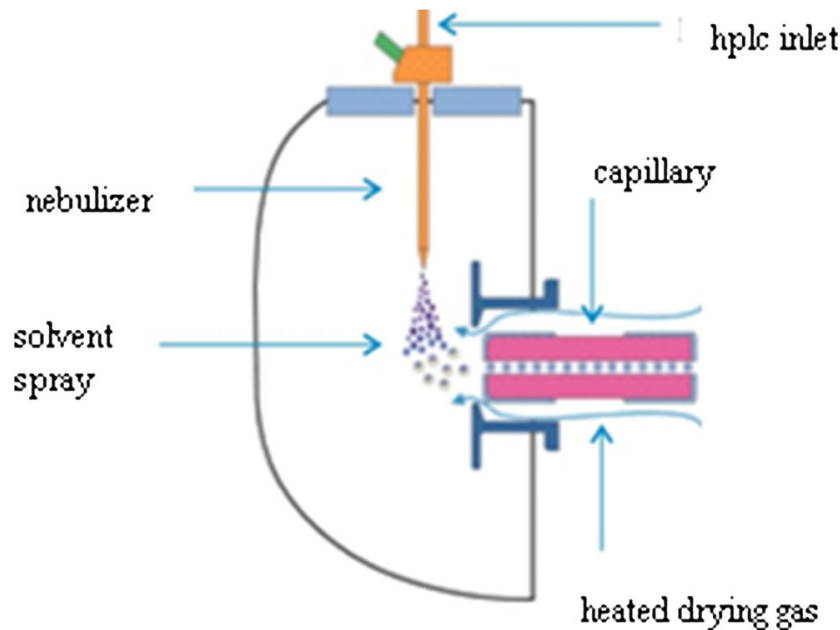

Fig. 1 Electrospray ionization (Conepts Guide-Agilent 6100 Series Quadrupole LC/MS Systems. Agilent Technologies 2010) frequently: quadrupole (Q), time-of-flight (TOF), and ion trap mass analyzer (IT).

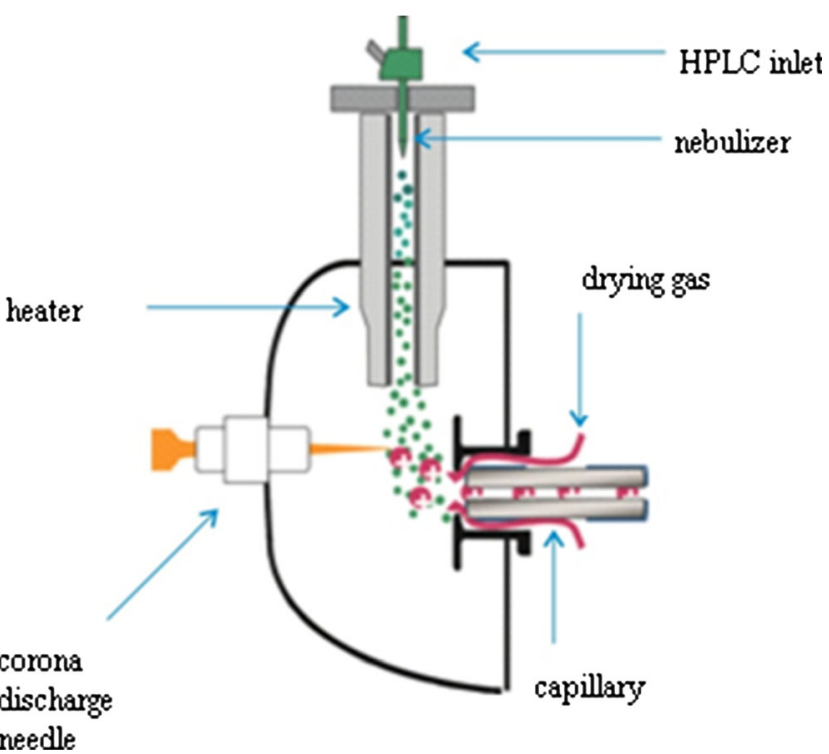

Fig. 2 Atmospheric pressure chemical ionization (Concepts GuideAgilent 6100 Series Quadrupole LC/MS Systems. Agilent Technologies 2010) 


\section{Quadrupole Analyzer}

The quadrupole analyzer $(\mathrm{Q})$ consists of four metal electrodes in the form of symmetrically arranged rods with a hyperbolic cross-section. It works like a mass filter that, with the specific parameters of the electromagnetic field, allows the passage of ions with the selected mass-to-charge ratio values, while other ions are dispersed and do not reach the detector (Johnstone and Malcolm 2001; Conepts Guide - Agilent 6100 Series Quadrupole LC/MS Systems. Agilent Technologies 2010).

The quadrupole analyzer is characterized by a relatively low separation efficiency that does not exceed 3000. Its separation efficiency is a function of ion mass and increases slightly as the mass increases. The quadrupole analyzer is a typical analyzer dedicated to the identification of ion mass. In the analysis of pesticide residue in food, it was used mainly in the first years of the period covered by the literature review, later it was gradually replaced by the most popular triple quadrupole mass analyzer used in tandem mass spectrometry (Romero-Gonzalez et al. 2006; Blasco et al. 2006; Liu et al. 2005; Frenich et al. 2005).

\section{Time-of-Flight Analyzer}

The time-of-flight mass analyzer (TOF) consists of an ion accelerating grid and a flight tube (about $1 \mathrm{~m}$ long), through which the ions travel to the detector. The analyzer separates ions accelerated by an electric field according to their velocity which depends on their mass and charge. The time-of-flight analyzer measures the time it takes an ion having a specific mass-to-charge ratio to reach the detector; the time is counted from the moment the ion is accelerated. The greater the massto-charge ratio of an ion, the longer it takes to reach the detector (Johnstone and Malcolm 2001).

The time-of-flight analyzer is characterized by a high scanning speed, broad measuring range, and high sensitivity. Its separation efficiency, up to 40,000 , considerably exceeds the separation efficiency of the quadrupole analyzer. In the analysis of pesticide residues in food, the time-of-flight analyzer is mainly used to confirm the presence of the compounds of interest in matrices such as fruit and vegetables (Mezcua et al. 2009; Taylor et al. 2008), honey and meat (Mol et al. 2008), fruit beverages (Gilbert-Lopez et al. 2012; GarciaReyes et al. 2008), and wine (Perez-Ortega et al. 2012).

\section{Ion Trap Analyzer}

One of the most popular ion trap analyzers (IT) is the quadrupole ion trap consisting of a ring-shaped electrode and two electrodes with a spherical cross-section, with the space between them forming a trap. The ion trap analyzer traps ions with a specific mass-to-charge ratio by means of an electric field. From the trap, the ions are sent to the detector, in the order of increasing $\mathrm{m} / \mathrm{z}$ values. The fragmentation of ions occurs through the collision of the charged ions with molecules of the neutral gas filling the ion trap. Alongside the quadrupole ion trap, there is also a linear ion trap whose structure is similar to the classical quadrupole analyzer. It also consists of four metal rods but, in the case of the ion trap, the electric potential unenabling the escape of the ions from the analyzer is placed at both ends of the analyzer (Johnstone and Malcolm 2001).

The range of analyzed masses and separation efficiency of ion traps (the latter reaching several thousands) are similar to those offered by quadrupole analyzers. Alongside the quadrupole analyzer, the ion trap analyzer is one of the most popular single MS analyzers in the analysis of pesticide residues in food (Tian 2011; Brutti et al. 2010; Radisic et al. 2009; Lesueur et al. 2008; Wang et al. 2007; Soler et al. 2007).

\section{Tandem Mass Spectrometry}

Tandem mass spectrometry (MS/MS) is a system of two combined analyzers of the same type or different types, characterized by high separation efficiency. The precursor ions produced by the source are separated in the first analyzer (MS1). Ions with the selected $\mathrm{m} / \mathrm{z}$ value reach the collision cell where, depending on the analysis conditions, they undergo dissociation or remain unchanged. Due to ion dissociation, referred to as fragmentation, product ions are generated and analyzed in the second analyzer (MS2) according to the numerical value of the $\mathrm{m} / \mathrm{z}$ parameter. Collision-induced dissociation (CID), involving the collision of ions with neutral gas molecules, is a fragmentation technique most frequently used in LC-MS/MS systems (Suder and Silberring 2006). In comparison with analysis using a single analyzer, tandem analysis shows a considerable improvement of the assay conditions through improved selectivity and considerably increased sensitivity. There are many possibilities of connecting different types of analyzers. Triple quadrupole systems (QQQ), quadrupole-time-of-flight systems (Q-TOF), and quadrupole-linear ion trap systems (Q-Trap) are the most frequent combinations of analyzers used in tandem mass spectrometry for the purpose of determining pesticide residues in food.

\section{Triple Quadrupole Tandem Mass Spectrometry}

The triple quadrupole (QQQ), where two quadrupoles function as ion analyzers and the third quadrupole in the middle serves as a collision cell, is one of the most popular tandem mass spectrometers. Only ions with a specific $\mathrm{m} / \mathrm{z}$ value, the socalled precursor ions, pass through the first quadrupole. These precursor ions are then subjected to fragmentation in the second quadrupole serving as the collision cell filled with inert gas. The fragmentation spectrum, i.e., the $m / z$ values of the product ions, is recorded by the detector; the spectrometer operates in 
product ion scan mode. When the third quadrupole allows only the selected product ions, the spectrometer works in the socalled multiple reaction monitoring mode (MRM). When the first quadrupole allows all ions formed in an ion source, the spectrometer is the full mass spectrum mode. When working in the full mass spectrum mode, the triple quadrupole system works on the same principle as the classic quadrupole analyzer. In a full spectrum measurement, the first two quadrupoles serve as beam collimators, and the entire analysis is carried out in the third quadrupole (Johnstone and Malcolm 2001).

Thanks to its high sensitivity and selectivity resulting from the possibility of the operation in the multiple reaction monitoring mode for the selected substances, the triple quadrupole tandem mass spectrometer is currently the most popular detector used in combination with a liquid chromatograph in the analysis of pesticide residues in food (Table 1).

\section{Quadrupole-Time-of-Flight Tandem Mass Spectrometer}

The quadrupole-time-of-flight (Q-TOF) tandem mass spectrometer is currently one of the most selective devices coupled with liquid chromatography. It is characterized by a very high separation efficiency. Similarly to QQQ, the principle of its operation is based on the series connection of quadrupoles where the third one is replaced by a time-of-flight analyzer. The precursor ions selected in the quadrupole analyzer are subjected to fragmentation in the collision cell, and the product ions thus generated travel at varying speeds through the time-of-flight analyzer and are recorded by the detector (Johnstone and Malcolm 2001).

First and foremost, the Q-TOF tandem mass spectrometer enables the determination of the elementary composition of the product ions generated as a result of the collision. Furthermore, based on the fragmentation of the ions analyzed, it is also possible to obtain structural information. The Q-TOF tandem mass spectrometer is used sporadically in the analysis of pesticide residues in food, mostly in order to obtain information on the scale of contamination by pesticides (BoteroCoy et al. 2012; Grimalt et al. 2010; Pico et al. 2009; Wang and Leung 2009; Soler et al. 2007).

\section{Quadrupole-Linear ion Trap Tandem Mass Spectrometer}

The quadrupole-linear ion trap tandem mass spectrometer (QTrap) combines the high sensitivity of the quadrupole with the capacity of the ion trap. This device is of the triple quadrupole type except that the third quadrupole is replaced by the linear ion trap. The ion selected in the quadrupole analyzer is fragmented in the ion trap. The advantage of this system is the fact that the ion trap can measure fragmentation spectra independently, and its series connection with another analyzer enables the recording of fragmentation spectra not only within MS/MS but also MS/MS/MS. The operation of ion trap-type devices is based on the accumulation of a large amount of ions which are then subjected to fragmentation and analysis. The whole process is periodic. The cycle takes place in one chamber and consists of the selection of the ion to be fragmented, collision with gas atoms/molecules, and analysis of the fragmented ions. In contrast to the tandem systems discussed above, the spectrometric analysis cycles in the ion trap are separated in time, not in space (Johnstone and Malcolm 2001).

In the analysis of pesticide residues in food, the quadrupole-linear ion trap tandem mass spectrometer has been used mainly to analyze pesticide residues in vegetables and fruit (Sinha et al. 2012; Afify et al. 2010; Lee et al. 2009a, b; Pihlstrom et al. 2007; Banerjee et al. 2007), (Lee et al. 2009a, b), cocoa beans (Zainudin et al. 2015), lavandin oil (Fillatre et al. 2014) fruit beverages (Tran et al. 2012; Ferrer et al. 2011), and fish muscle (Lazartigues et al. 2011).

\section{Ion Recording Techniques}

Depending on their construction and analysis purpose, mass spectrometers can work in various ion monitoring modes. The mass spectrum is the direct result of their operation. In the mass spectra of positive and negative ions, one can observe, respectively, ions of the protonated molecules $[\mathrm{M}+\mathrm{H}]^{+}$and deprotonated molecules $[\mathrm{M}-\mathrm{H}]^{-}$, corresponding to the molecular mass of substances. Furthermore, in mass spectra, one can also observe ions that are products of the binding of, for example, an ammonium ion or alkali metal ions such as $[\mathrm{M}+$ $\left.\mathrm{NH}_{4}\right]^{+},[\mathrm{M}+\mathrm{Na}]^{+}$, or $[\mathrm{M}+\mathrm{K}]^{+}$in conditions of positive ion formation, while $[\mathrm{M}+\mathrm{HCOO}]^{-}$or $[\mathrm{M}+\mathrm{CH} 3 \mathrm{COO}]^{-}$ions form in conditions of negative ion formation, in the presence of formic acid and/or ammonium formate or acetic acid and/or ammonium acetate. The possibility of the ionization of molecules of an individual substance through the binding of various ions leads to a significant reduction of sensitivity but, on the other hand, the presence of all the abovementioned ion types in the mass spectrum makes it possible to confirm the molecular mass of the compound of interest present in the sample (Suder and Silberring 2006). The fragmentation stage is necessary to determine the structure of the compound besides the molecular mass. The ions of the protonated molecules $[\mathrm{M}+\mathrm{H}]^{+}$formed through ionization or other ions generated in the source are subjected to fragmentation through collision-induced dissociation. The product ions, depending on their $\mathrm{m} / \mathrm{z}$ value, are separated in the analyzer, from which they move to the detector that transforms the ion current into the measurable analytical signal (Suder and Silberring 2006). The recorded mass spectra of the fragmented ions provide structural information about a specific compound. Regardless of the sample ionization method used, three main ion recording methods are distinguished (Czerwicka et al. 2012): 
- Recording of total ion current (TIC), also referred to as full scan, scanning, or reconstructed ion chromatogram (RIC) offering the full mass spectrum from the preset $\mathrm{m} / \mathrm{z}$ range

- Selected ion monitoring (SIM) or single ion recording (SIR) enabling the recording of the selected ions, characteristic of the selected compounds

- Multiple reaction monitoring (MRM), selected reaction monitoring (SRM), enabling the confirmation of the presence of fragmented ions formed from a specific precursor ion

The ion recording methods above vary not only with regard to the technique used to obtain analytical information but also the range of selectivity and sensitivity. The Selected Ion Monitoring technique (for ions characteristic of specific compounds) shows a considerably greater selectivity and sensitivity than the total ion current technique that records all ions originating from a specific chemical compound. The multiple reaction monitoring technique is characterized by the greatest sensitivity. Furthermore, the high level of selectivity of MRM makes it one of the most reliable methods for confirming the presence of a specific compound in the sample. Summing up, the sensitivity and selectivity of the ion recording methods increases in the following order: TIC $<$ SIM $<$ MRM. However, SIM and MRM techniques are only used to analyze compounds whose mass spectra and fragmentation paths are well known. In the case of unknown compounds, the TIC technique is used (Czerwicka et al. 2012).

Multiple reaction monitoring has been the most frequently used technique of ion recording in the LC-MS analysis of pesticide residues in food. The share of the other monitoring techniques is shown in Fig. 3.

\section{Matrix Effect}

Thanks to the wide range of analyzers and operation modes, liquid chromatography coupled with mass spectrometry (LCMS) or tandem mass spectrometry (LC-MS/MS) enables the

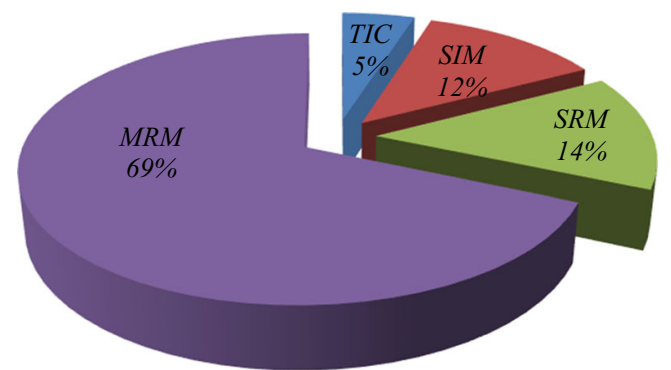

Fig. 3 Comparison of the usage frequency of the individual ion monitoring techniques used in the LC-MS analysis of pesticide residues in food samples (notation explained in the text). According to the Scopus database, keywords: pesticide, food, multiresidue methods, LC-MS trace analysis of a wide range of compounds. Its main advantages include very high sensitivity, understood as the low limit of quantification, and high selectivity enabling the determination of the analyte in complex mixtures.

The main limitation of the LC-MS technique is the sensitivity to the accompanying matrix components. Exo- and endogenic substances present in the sample can interfere with the analyte in the ion source, resulting in an increased or reduced ionization of the analyte. This effect leads to the suppression or, much more rarely, enhancement of the analytical signal. The matrix effect is considerably more distinct for more complex matrices. To reduce the matrix effect and/or calibrate its impact on the result of the analysis, the following are used at the stage of preparing the sample for investigation: matrix-matched calibration, internal standard addition (most often an isotope-labeled analyte analogue), or increasing the intensity of extract purification; while changing the chromatographic conditions (of the stationary and/or mobile phase) and extending the duration of the analysis are the methods used at the stage of instrumental analysis. Good laboratory practice in trace analysis recommends the use of pre-columns whose task is to purify the sample from contaminants that reduce the performance of the ion source.

Calibration using the sample matrix is the most popular: it is based on preparing calibration solutions containing components of the sample matrix rather than a pure solvent. Analytical signals coming from the analytes present in the sample analyzed are compared to those present in the solutions used for calibration. The limitations of calibration using the sample matrix result from the difficulty of obtaining a sample matrix free of the analyte and, when analyzing a large number of samples with varied matrices, the need to conduct a separate calibration for each matrix, which requires greater amounts of standards of the substances analyzed, and is very time-consuming and labor-intensive. Therefore, using a single representative matrix to prepare calibration solutions is often permitted for the same group of products with a similar matrix composition (Gómez-Pérez et al. 2015; Stachniuk and Fornal 2013; Fernandez-Alba and Garcia-Reyes 2008; Vidal et al. 2004).

According to the internal standard (IS) addition method, an appropriate standard whose physicochemical properties are similar to the compounds determined is added to the solution of the sample analyzed. Internal standard addition is particularly recommended for multiresidue analysis (Vidal et al. 2004). For the internal standard to adjust the matrix effect, it has to coelute with the analyte. Therefore, isotope-labeled analyte analogues are most often used as internal standards. Unfortunately, the use of these analogues raises the costs of analysis considerably.

Increasing the intensity of extract purification is frequently used as a method of matrix effect reduction. Intensive purification of the extract considerably reduces the matrix effect but 


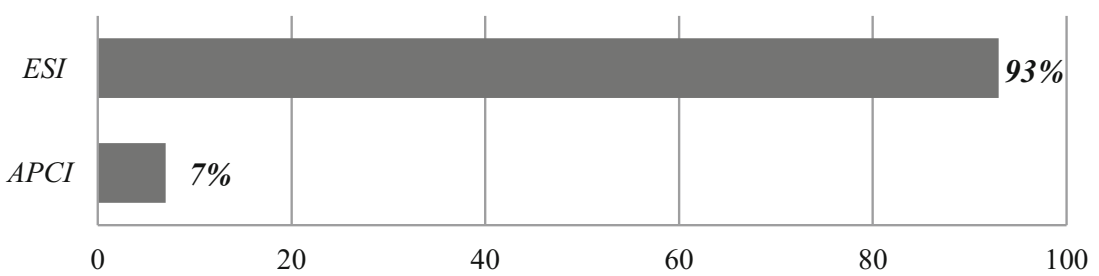

Fig. 4 Comparison of the usage frequency of the individual ionization techniques used in the LC-MS analysis of pesticide residues in food samples (notation explained in the text). According to the Scopus database, keywords: pesticide, food, multiresidue methods, LC-MS

can also cause losses of the analyzed compounds and increased analysis cost and duration. The opposite, i.e., an insufficient purification of the extract can have a negative impact on the quality of the results.

In order to reduce the matrix effect and increase the accuracy of determination in the analysis of pesticide residues in a broad range of food matrices, calibration using the representative or food-specific matrix-matching calibration along with the internal standard addition is usually conducted at the sample preparation stage.

Two other simple solutions to overcome or compensate matrix effect are also often applied: the sample dilution and the standard addition method. Since the latest models of LCMS/MS spectrometers offer very high sensitivity, the sample dilution with solvent is used nowadays in many laboratories as the simplest way of the matrix effect reduction. In the standard addition method, the sample is divided into two portions. To one portion, the targeted pesticide is added, usually at twofold concentration detected, and the same volume of solvent is added to the other. The pesticide concentration in the sample is then extrapolated with the two point calibration line.

\section{Conclusions and Final Remarks}

The coupling of the effective liquid chromatography separation technique with the selective mass spectrometry detection technique has revolutionized the analytical possibilities of quantification, enabling the detection of an increasing number of compounds in a single determination as well as ever smaller trace amounts of substances in the complex sample analyzed. Several kinds of couplings of liquid chromatography with a mass spectrometer are currently available and each has different applications and requires devices of different construction. Given the broad range of its applications, the electrospray ionization technique (ESI) has become the dominant kind of ionization since its introduction into the market. The popularity of ESI is reflected in its usage frequency shown in Fig. 4.

As technology has advanced, new designs of mass spectrometers have appeared in the market, differing not only with regard to ionization methods but also the kind of analyzers. Modern mass spectrometers often enable a quick change of the ion source and combine multiple analyzers, thanks to which it is possible to dynamically switch between MS and
MS/MS modes. Selecting the optimum MS device for a specific analysis depends on the kind of product analyzed as well as the purpose of the analysis: whether it is a quantitative or qualitative analysis, and whether we want to determine the mass of the analyte only or its structure as well.

Nowadays, a high-performance liquid chromatograph coupled with a QQQ tandem mass spectrometer, working in the multiple reaction monitoring (MRM) mode, is the most frequently used platform used in the analysis of pesticide residues in food. Figure 5 compares the types of mass spectrometers used in the analysis of pesticide residues in food in recent years.

Alongside the most popular quadrupole MS and MS/MS systems, the quadrupole-ion trap system is most frequently used in the analysis of pesticide residues in food. Time-offlight analyzers are used relatively rarely.

The most important advantages of high-performance/ultraperformance liquid chromatography interfaced with a mass spectrometer include high sensitivity and selectivity, short duration of analysis, which enables the separation and determination of a considerable number of compounds during a single analytical cycle. This is why this technique is so popular in multiresidue analysis of various substances, including pesticides. The main limitation of the technique is its sensitivity to the accompanying matrix components, particularly in the analysis of compounds occurring in complex biological matrices, e.g., in food. Calibration using a sample matrix is the

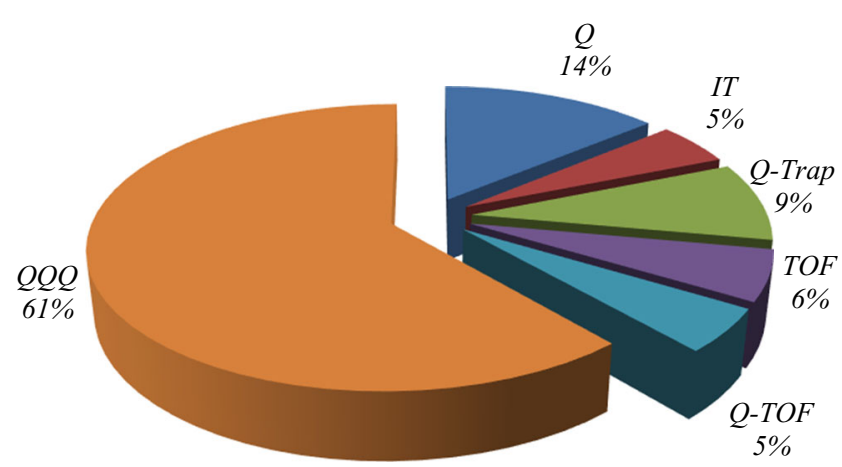

Fig. 5 Comparison of the usage frequency of the individual analyzers and tandem systems interfaced with liquid chromatography and used in the analysis of pesticide residues in food samples (notation explained in the text). According to the Scopus database, keywords: pesticide, food, multiresidue methods, LC-MS 
most frequent technique used to reduce the matrix effect on the quantification result.

The analysis of pesticide residues in food is subject to constant modification owing to matrix complexity, low concentrations of the compounds of interest, and increasing number of pesticides approved for use. Thanks to the development of test equipment, scientific laboratories continue to develop new analytical methodologies, making it possible to lower the detection limit and expand the scope of the existing methods to include new compounds. A significant role is played by the analyst whose experience in operating LC-MS and LC-MS/ MS systems can have a considerable impact on the quality and scientific value of the procedures developed.

\section{Compliance with Ethical Standards}

Conflict of Interest Author A.M. Stachniuk declares that she has no conflict of interest. Author E. Fornal declares that she has no conflict of interest.

Ethical Approval This article does not contain any studies with human participants or animals performed by any of the authors.

Informed Consent Not applicable.

Open Access This article is distributed under the terms of the Creative Commons Attribution 4.0 International License (http:// creativecommons.org/licenses/by/4.0/), which permits unrestricted use, distribution, and reproduction in any medium, provided you give appropriate credit to the original author(s) and the source, provide a link to the Creative Commons license, and indicate if changes were made.

\section{References}

Afify AEMM, Mohamed MA, El-Gammal HA, Attallah ER (2010) Multiresidue method of analysis for determination of 150 pesticides in grapes using quick and easy method (QuEChERS) and LC-MS/ MS determination. J Food Agric Environ 8:602-606

Aguilera-Luiz M, Plaza Bolanos P, Romero-Gonzalez R, Martinez Vidal J, Frenich A (2011) Comparison of the efficiency of different extraction methods for the simultaneous determination of mycotoxins and pesticides in milk samples by ultra high-performance liquid chromatography-tandem mass spectrometry. Anal Bioanal Chem 399:2863-2875

Alder L, Greulich K, Kempe G, Vieth B (2006) Residue analysis of 500 high priority pesticides: better by GC-MS or LC-MS/MS? Mass Spectrom Rev 25:838-865

Anagnostopoulos C, Aplada Sarli P, Liapis K, Haroutounian S, Miliadis $G$ (2012) Validation of two variations of the QuEChERS method for the determination of multiclass pesticide residues in cereal-based infant foods by LC-MS/MS. Food Anal Method 5:664-683

Arienzo M, Cataldo D, Ferrara L (2013) Pesticide residues in fresh-cut vegetables from integrated pest management by ultra-performance liquid chromatography coupled to tandem mass spectrometry. Food Control 31:108-115

Banerjee K, Oulkar DP, Dasgupta S, Patil SB, Patil SH, Savant R, Adsule PG (2007) Validation and uncertainty analysis of a multi-residue method for pesticides in grapes using ethyl acetate extraction and liquid chromatography-tandem mass spectrometry. J Chromatogr A 1173:98-109

Blasco C, Font G, Pico Y (2006) Evaluation of 10 pesticide residues in oranges and tangerines from Valencia (Spain). Food Control 17: 841-846

Bogialli S, Curini R, Di Corcia A, Lagana A, Stabile A, Sturchio E (2006) Development of a multiresidue method for analyzing herbicide and fungicide residues in bovine milk based on solid-phase extraction and liquid chromatography-tandem mass spectrometry. J Chromatogr A 1102:1-10

Botero-Coy AM, Marin JM, Ibanez M, Sancho JV, Hernandez F (2012) Multi-residue determination of pesticides in tropical fruits using liquid chromatography/tandem mass spectrometry. Anal Bioanal Chem 402:2287-2300

Botitsi HV, Garbis SD, Economou A, Tsipi DF (2011) Current mass spectrometry strategies for the analysis of pesticides and their metabolites in food and water matrices. Mass Spectrom Rev 30:907939

Brutti M, Blasco C, Pico Y (2010) Determination of benzoylurea insecticides in food by pressurized liquid extraction and LC-MS. J Sep Sci 33:1-10

Camino-Sanchez FJ, Zafra-Gomez A, Oliver-Rodriguez B, Ballestero O, Navalon A, Crovetto G, Vilchez JL (2010) UNE-EN ISO/IEC 17025:2005-accredited method for the determination of pesticide residues in fruit and vegetable samples by LC-MS/MS. Food Addit Contam A 27:1532-1544

Carmona J, Steininger D, Cole J, Silcock P, Beck J, Blackburn M, Massi J, Yang C, Ghosh D (2013) Comparing LC and GC Triple Quadrupole MS for the screening of 500 pesticides in matrix, 61st Annual ASMS Conference on Mass Spectrometry and Allied Topics, June 9 - 13, 2013, Minneapolis, MN, USA, session: Food safety - pesticides THP27, poster number: 544, available from https://www.thermoscientific.com/content/dam/tfs/ATG/CMD/ CMD\%20Documents/PN-Screening-500-Pesticides-MatrixASMS13-Th544-JCole-EN.pdf

Carneiro RP, Oliveira FAS, Madureira FD, Silva G, de Souza WR, Lopes RP (2013) Development and method validation for determination of 128 pesticides in bananas by modified QuEChERS and UHPLCMS/ MS analysis. Food Control 33:413-423

Chen GQ, Cao PY, Liu RJ (2011) A multi-residue method for fast determination of pesticides in tea by ultra performance liquid chromatography-electrospray tandem mass spectrometry combined with modified QuEChERS sample preparation procedure. Food Chem 125:1406-1411

Choi S, Kim S, Shin JY, Kim MK, Kim JH (2015) Development and verification for analysis of pesticides in eggs and egg products using QuEChERS and LC-MS/MS. Food Chem 173:1236-1242

Chung SWC, Lam CH (2012) Development and validation of a method for determination of residues of 15 pyrethroids and two metabolites of dithiocarbamates in foods by ultra-performance liquid chromatography-tandem mass spectrometry. Anal Bioanal Chem 403:885-896

Conepts Guide - Agilent 6100 Series Quadrupole LC/MS Systems. Agilent Technologies 2010

Czerwicka M, Kumirska J, Stepnowski P (2012) Spektrometria masuniwersalna technika analityczna (paper in Polish) (mass spectrometry-universal analytical technique). Laboratorium 5-6: $20-22$

Economou A, Botitsi H, Antoniou S, Tsipi D (2009) Determination of multi-class pesticides in wines by solid-phase extraction and liquid chromatography-tandem mass spectrometry. J Chromatogr A 1216: 5856-5867

Esturk O, Yakar Y, Ayhan Z (2014) Pesticide residue analysis in parsley, lettuce and spinach by LC-MS/MS. J Food Sci Tech Mys 51:458466 
Fernandez-Alba AR, Garcia-Reyes JF (2008) Large-scale multi-residue methods for pesticides and their degradation products in food by advanced LC-MS. Trac-Trend Anal Chem 27:973-990

Ferrer C, Martinez-Bueno MJ, Lozano A, Fernandez-Alba AR (2011) Pesticide residue analysis of fruit juices by LC-MS/MS direct injection. One year pilot survey. Talanta 83:1552-1561

Fillatre Y, Rondeau D, Daguin A, Jadas-Hecart A, Communal PY (2014) Multiresidue determination of 256 pesticides in lavandin essential oil by LC/ESI/sSRM: advantages and drawbacks of a sampling method involving evaporation under nitrogen. Anal Bioanal Chem 406:1541-1550

Frenich AG, Salvador IM, Martinez Vidal J, Lopez-Lopez T (2005) Determination of multiclass pesticides in food commodities by pressurized liquid extraction using GC-MS/MS and LC-MS/MS. Anal Bioanal Chem 383:1106-1118

Garcia-Reyes JF, Gilbert-Lopez B, Molina-Diaz A, Fernandez-Alba AR (2008) Determination of pesticide residues in fruit-based soft drinks. Anal Chem 80:8966-8974

Gilbert-Lopez B, Jaen-Martos L, Garcia-Reyes JF, Villar-Pulido M, Polgar L, Ramos-Martos N, Molina-Diaz A (2012) Study on the occurrence of pesticide residues in fruit-based soft drinks from the EU market and morocco using liquid chromatography-mass spectrometry. Food Control 26:341-346

Golge O, Kebak B (2015) Evaluation of QuEChERS sample preparation and liquid chromatography-triple-quadrupole mass spectrometry method for the determination of 109 pesticide residues in tomatoes. Food Chem 176:319-332

Gómez-Pérez ML, Romero-González R, Vidal JLM, Frenich AG (2015) Analysis of pesticide and veterinary drug residues in baby food by liquid chromatography coupled to Orbitrap high resolution mass spectrometry. Talanta 131:1-7

González-Curbelo MA, Lehotay SJ, Hernández-Borges J, RodríguezDelgado MA (2014) Use of ammonium formate in QuEChERS for high-throughput analysis of pesticides in food by fast, lowpressure gas chromatography and liquid chromatography tandem mass spectrometry. J Chromatogr A 1358:75-84

Grimalt S, Sancho JV, Pozo OJ, Hernandez F (2010) Quantification, confirmation and screening capability of UHPLC coupled to triple quadrupole and hybrid quadrupole time-of-flight mass spectrometry in pesticide residue analysis. J Mass Spectrom 45:421-436

Hernandez F, Pozo OJ, Sancho JV, Bijlsma L, Barreda A, Pitarch E (2006) Multiresidue liquid chromatography tandem mass spectrometry determination of 52 non gas chromatography-amenable pesticides and metabolites in different food commodities. J Chromatogr A 1109:242-252

Hildmann F, Gottert C, Frenzel T, Guenther K, Speer K (2015) Pesticide residues in chicken eggs - a sample preparation methodology for analysis by gas and liquid chromatography/tandem mass spectrometry. J Chromatogr A 1403:1-20

Jardim ANO, Mello DC, Goes FCS, Junior EFF, Caldas ED (2014) Pesticide residues in cashew apple, guava, kaki and peach: GCIECD, GC-FPD and LC-MS/MS multiresidue method validation, analysis and cumulative acute risk assessment. Food Chem 164: 195-204

Johnstone R, Malcolm R (2001) Spektrometria mas (book in Polish) (mass spectrometry). PWN, Warszawa

Kamel A, Qian YR, Kolbe E, Stafford C (2010) Development and validation of a multiresidue method for the determination of neonicotinoid and macrocyclic lactone pesticide residues in milk, fruits, and vegetables by ultra-performance liquid chromatography/ MS/MS. J Aoac Int 93:389-399

Kmellar B, Fodor P, Pareja L, Ferrer C, Martinez-Uroz MA, Valverde A, Fernandez-Alba AR (2008) Validation and uncertainty study of a comprehensive list of 160 pesticide residues in multi-class vegetables by liquid chromatography-tandem mass spectrometry. J Chromatogr A 1215:37-50
Kmellar B, Pareja L, Ferrer C, Fodor P, Fernandez-Alba AR (2011) Study of the effects of operational parameters on multiresidue pesticide analysis by LC-MS/MS. Talanta $84: 262-273$

Kujawski MW, Barganska Z, Marciniak K, Miedzianowska E, Kujawski JK, Slebioda M, Namiesnik J (2014) Determining pesticide contamination in honey by LC-ESI-MS/MS - comparison of pesticide recoveries of two liquid-liquid extraction based approaches. LWTFood Sci Technol 56:517-523

Lazartigues A, Wiest L, Baudot R, Thomas M, Feidt C, Cren-Olive C (2011) Multiresidue method to quantify pesticides in fish muscle by QuEChERS-based extraction and LC-MS/MS. Anal Bioanal Chem 400:2185-2193

Lee SJ, Park S, Choi JY, Shim JH, Shin EH, Choi JH, Kim ST, El-Aty AMA, Jin JS, Bae DW, Shin SC (2009a) Multiresidue analysis of pesticides with hydrolyzable functionality in cooked vegetables by liquid chromatography tandem mass spectrometry. Biomed Chromatogr 23:719-731

Lee SJ, Park HJ, Kim W, Jin JS, El-Aty AMA, Shim JH, Shin SC (2009b) Multiresidue analysis of 47 pesticides in cooked wheat flour and polished rice by liquid chromatography with tandem mass spectrometry. Biomed Chromatogr 23:434-442

Lee SW, Choi JH, Cho SK, Yu HA, Abd El-Aty AM, Shim JH (2011) Development of a new QuEChERS method based on dry ice for the determination of 168 pesticides in paprika using tandem mass spectrometry. J Chromatogr A 1218:4366-4377

Lee H, Park SS, Lim MS, Lee HS, Park HJ, Hwang HS, Park SY, Cho DH (2013) Multiresidue analysis of pesticides in agricultural products by a liquid chromatography/tandem mass spectrometry based method. Food Sci Biotechnol 22:1205-1216

Lehotay SJ, Mastovska K, Lightfield AR (2005) Use of buffering and other means to improve results of problematic pesticides in a fast and easy method for residue analysis of fruits and vegetables. J Aoac Int 88:615-629

Lehotay SJ, Mastovska K, Lightfield AR, Gates RA (2010) Multi-analyst, multi-matrix performance of the QuEChERS approach for pesticide residues in foods and feeds using HPLC/MS/MS analysis with different calibration techniques. J Aoac Int 93:355-367

Lesueur C, Knittl P, Gartner M, Mentler A, Fuerhacker M (2008) Analysis of 140 pesticides from conventional farming foodstuff samples after extraction with the modified QuECheRS method. Food Control 19:906-914

Liu M, Hashi Y, Song YY, Lin JM (2005) Simultaneous determination of carbamate and organophosphorus pesticides in fruits and vegetables by liquid chromatography-mass spectrometry. J Chromatogr A 1097:183-187

Lozano A, Rajski L, Belmonte-Valles N, Ucles A, Ucles S, Mezcua M, Fernandez-Alba AR (2012) Pesticide analysis in teas and chamomile by liquid chromatography and gas chromatography tandem mass spectrometry using a modified QuEChERS method: validation and pilot survey in real samples. J Chromatogr A 1268:109-122

Martinez-Dominguez G, Nieto- Garcia AJ, Romero-Gonzalez R, Frenich AG (2015) Application of QuEChERS based method for the determination of pesticides in nutraceutical products (Camellia sinensis) by liquid chromatography coupled to triple quadrupole tandem mass spectrometry. Food Chem 177:182-190

Mastovska K, Dorweiler KJ, Lehotay SJ, Wegscheid JS, Szpylka KA (2010) Pesticide multiresidue analysis in cereal grains using modified QuEChERS method combined with automated direct sample introduction GC-TOFMS and UPLC-MS/MS techniques. J Agr Food Chem 58:5959-5972

Mezcua M, Malato O, Garcia-Reyes JF, Molina-Diaz A, Fernandez-Alba AR (2009) Accurate-mass databases for comprehensive screening of pesticide residues in food by fast liquid chromatography time-offlight mass spectrometry. Anal Chem 81:913-929

Mol HGJ, Plaza-Bolanos P, Zomer P, de Rijk TC, Stolker AAM, Mulder PPJ (2008) Toward a generic extraction method for simultaneous 
determination of pesticides, mycotoxins, plant toxins, and veterinary drugs in feed and food matrixes. Anal Chem 80:9450-9459

Nozal J, Bernal JL, Jimenez JJ, Martin T, Bernal J (2005) Determination of azolic fungicides in wine by solid-phase extraction and highperformance liquid chromatography-atmospheric pressure chemical ionization-mass spectrometry. J Chromatogr A 1076:90-96

Nunez O, Gallart-Ayala H, Ferrer I, Moyano E, Galceran MT (2012) Strategies for the multi-residue analysis of 100 pesticides by liquid chromatography-triple quadrupole mass spectrometry. J Chromatogr A 1249:164-180

Omate M, Harayama K, Sasaki T, Mochizuki N, Yamashita H (2006) Analysis of simultaneous screening for 277 pesticides in malt and beer by liquid chromatography with tandem mass spectrometry. J Am Soc Brew Chem 64:139-150

Oshita D, Jardim ICSF (2014) Comparison of different sorbents in the QuEChERS method for the determination of pesticide residues in strawberries by LC-MS/MS. Chromatographia 77:1291-1298

Pareja L, Fernandez-Alba AR, Cesio V, Heinzen H (2011) Analytical methods for pesticide residues in rice. Trac-Trend Anal Chem 30: 270-291

Perez-Ortega P, Gilbert-Lopez B, Garcia-Reyes JF, Ramos-Martos N, Molina-Diaz A (2012) Generic sample treatment method for simultaneous determination of multiclass pesticides and mycotoxins in wines by liquid chromatography-mass spectrometry. J Chromatogr A 1249:32-40

Pico Y, Blasco C, Farre M, Barcelo D (2009) Analytical utility of quadrupole time-of-flight mass spectrometry for the determination of pesticide residues in comparison with an optimized column highperformance liquid chromatography/tandem mass spectrometry method. J Aoac Int 92:734-744

Pihlstrom T, Blomkvist G, Friman P, Pagard U, Osterdahl BG (2007) Analysis of pesticide residues in fruit and vegetables with ethyl acetate extraction using gas and liquid chromatography with tandem mass spectrometric detection. Anal Bioanal Chem 389:1773-1789

Pizzutti IR, de Kok A, Zanella R, Adaime MB, Hiemstra M, Wickert C, Prestes OD (2007) Method validation for the analysis of 169 pesticides in soya grain, without clean up, by liquid chromatographytandem mass spectrometry using positive and negative electrospray ionization. J Chromatogr A 1142:123-136

Radisic M, Grujic S, Vasiljevic T, Lausevic M (2009) Determination of selected pesticides in fruit juices by matrix solid-phase dispersion and liquid chromatography-tandem mass spectrometry. Food Chem 113:712-719

Raina R (2011) Chemical analysis of pesticides using GC/MS, GC/MS/ MS, and LC/MS/MS, Pesticides Strategies for Pesticides Analysis, Prof. Margarita Stoytcheva (Ed.), ISBN: 978-953-307-460-3, InTech, 105-130, available from: http://www.intechopen.com/ books/pesticides-strategies-for-pesticides-analysis/chemicalanalysis-of-pesticides-using-GC-MS-GC-MS-MS-and-LC-MS-MS

Rajski Ł, Lozano A, Uclés A, Ferrer C, Fernández-Alba AR (2013) Determination of pesticide residues in high oil vegetal commodities by using various multi-residue methods and clean-ups followed by liquid chromatography tandem mass spectrometry. J Chromatogr A 1304:109-120

Romero-Gonzalez R, Pastor-Montoro E, Martinez-Vidal JL, GarridoFrenich A (2006) Application of hollow fiber supported liquid membrane extraction to the simultaneous determination of pesticide residues in vegetables by liquid chromatography/mass spectrometry. Rapid Commun Mass Sp 20:2701-2708

Sannino A, Bandini M (2005) Determination of seven benzoylphenylurea insecticides in processed fruit and vegetables using highperformance liquid chromatography/tandem mass spectrometry. Rapid Commun Mass 19:2729-2733
Silva RO, Carius de Castro R, Milhome Liberato MA, Ferreira do Nasciment R (2014) Liquid chromatography-electrospray ionization-tandem mass spectrometry method for determination of twenty multi-class pesticide residues in cashew. LWT- Food Sci Technol 59: $21-25$

Sinha SN, Vasudev K, Rao MVV (2012) Quantification of organophosphate insecticides and herbicides in vegetable samples using the "quick easy cheap effective rugged and safe" (QuEChERS) method and a high-performance liquid chromatography-electrospray ionisation-mass spectrometry (LC-MS/MS) technique. Food Chem 132:1574-1584

Sivaperumal P, Anand P, Riddhi L (2015) Rapid determination of pesticide residues in fruits and vegetables, using ultra-high-performance liquid chromatography/time-of-flight mass spectrometry. Food Chem 168:356-365

Soler C, James KJ, Pico Y (2007) Capabilities of different liquid chromatography tandem mass spectrometry systems in determining pesticide residues in food. Application to estimate their daily intake. J Chromatogr A 1157:73-84

Stachniuk A, Fornal E (2013) Analytical considerations on the use of a fruit-specific and representative matrix in pesticide residue analysis by LC-ESI-MS/MS. Cent Eur J Chem 11:1112-1131

Suder P, Silberring J (2006) Spektrometria mas (book in Polish) (mass spectrometry). Wydawnictwo UJ, Kraków

Swartz ME (2005) UPLC: an introduction and review. J LiqChromatogr R T 28:1253-1263

Taylor MJ, Keenan GA, Reid KB, Fernandez DU (2008) The utility of ultra-performance liquid chromatography/electrospray ionisation time-of-flight mass spectrometry for multi-residue determination of pesticides in strawberry. Rapid Commun Mass Sp 22:2731-2746

Tian HZ (2011) Determination of chloramphenicol, enrofloxacin and 29 pesticides residues in bovine milk by liquid chromatographytandem mass spectrometry. Chemosphere 83:349-355

Tran K, Eide D, Nickols SM, Cromer MR, Sabaa-Srur A, Smith RE (2012) Finding of pesticides in fashionable fruit juices by LC-MS/ MS and GC-MS/MS. Food Chem 134:2398-2405

Tseng SH, Liu CC, Lin YJ, Chen HC, Su SC, Chou HK, Chou SS, Shih DYC (2009) Analysis of 81 pesticides and metabolite residues in fruits and vegetables by diatomaceous earth column extraction and LC/MS/MS determination. J Food Drug Anal 17:319-332

Vidal JLM, Arrebola FJ, Frenich AG, Ferníndez JM, Mateu-Sanchez M (2004) Validation of a gas chromatographic tandem mass spectrometric method for analysis of pesticide residues in six food commodities. Selection of a reference matrix for calibration. Chromatographia 59:321-327

Wang J, Leung D (2009) Applications of ultra-performance liquid chromatography electrospray ionization quadrupole time-of-flight mass spectrometry on analysis of 138 pesticides in fruit- and vegetablebased infant foods. J Agr Food Chem 57:2162-2173

Wang SL, Xu YJ, Pan CP, Jiang S, Liu FM (2007) Application of matrix solid-phase dispersion and liquid chromatography-mass spectrometry to fungicide residue analysis in fruits and vegetables. Anal Bioanal Chem 387:673-685

Zainudin BH, Salleh S, Mohamed R, Yap KC, Muhamed H (2015) Development, validation and determination of multiclass pesticide residues in cocoa beans using gas chromatography and liquid chromatography tandem mass spectrometry. Food Chemi 172:585-595

Zhang XA, Mobley N, Zang JG, Zheng XM, Lu L, Ragin O, Smith CJ (2010) Analysis of agricultural residues on tea using d-SPE sample preparation with GC-NCI-MS and UHPLC-MS/MS. J Agr Food Chem 58:11553-11560 